\title{
Vibrational Structure of Organic Semiconductors: The Role of Conjugation Length
}

\author{
C. A. M. Borges, A. Marletta ${ }^{\dagger}$, R. M. Faria, and F. E. G. Guimarães \\ Instituto de Física de São Carlos, Universidade de São Paulo, CP 369, CEP 13560-970, São Carlos, SP, Brazil \\ ${ }^{\dagger}$ Faculdade de Física, Universidade Federal de Uberlândia, CP 593, CEP 38400-902, Uberlândia, MG, Brazil
}

Received on 31 March, 2003

\begin{abstract}
Site-selective luminescence spectroscopy (SSL) was employed to resolve the vibracional modes in selfassembled poly( $p$-phenylene vinylene), PPV. Using Franck-Condon analysis, the PPV spectra can be well described by three effective vibrational modes at 330,1160 and $1550 \mathrm{~cm}^{-1}$. As the temperature increases from 4 to $180 \mathrm{~K}$ the electron-phonon coupling ( $\mathrm{S}$ factor) remains constant for the $1550 \mathrm{~cm}^{-1}$ mode while for other lower energy modes the coupling increases monotonically. This trend is consistent with the decrease of the conjugation length due to the increase of thermal disorder. We find that the temperature dependence of vibrational progression in PPV films is determined mainly by low frequency torsional modes.
\end{abstract}

\section{Introduction}

The coupling of the electronic properties to structural changes is of special importance in the case of conjugated polymers [1-3]. The parameter that makes optical properties to be very sensitive to conformational and thermal disorder is the Huang-Rhys factor $S$. The coupling constant $\mathrm{S}$ is related to disorder through the degree of the chain conjugation. However, the exact relation between S-factor and conjugation length is not well establish [3]. In the present work, we studied experimentally and theoretically the effects of thermal disorder on electron-phonon coupling in the emission spectra of the model polymer poly ( $p$-phenylene vinylene), PPV.

\section{Experimental procedure}

The experiments were performed with high quality PPV films prepared by self-assembly (SA) methodology and converted at $110{ }^{\circ} \mathrm{C}$ for $30 \mathrm{~min}$ [5]. Site-selective luminescence (SSL) was carried out using a very narrow excitationline (spectral bandwidth of $\sim 1 \mathrm{~nm}$ ), which was obtained through the dispersion of the light of a $450 \mathrm{~W}$ Xe-lamp by a $1 \mathrm{~m}$ monochromator. Details of the PL set-up are given in Ref. [5].

\section{Results and discussions}

Figure 1 displays luminescence spectra $(40 \mathrm{~K})$ of a SA PPV film upon scanning the light excitation wavelength $\lambda_{\text {exc }}$ across the low energy tail of the absorption band that overlaps the luminescence spectrum. In the non-resonant case $\left(\lambda_{e x c}=495 \mathrm{~nm}\right)$ we observed the standard PPV spectra with

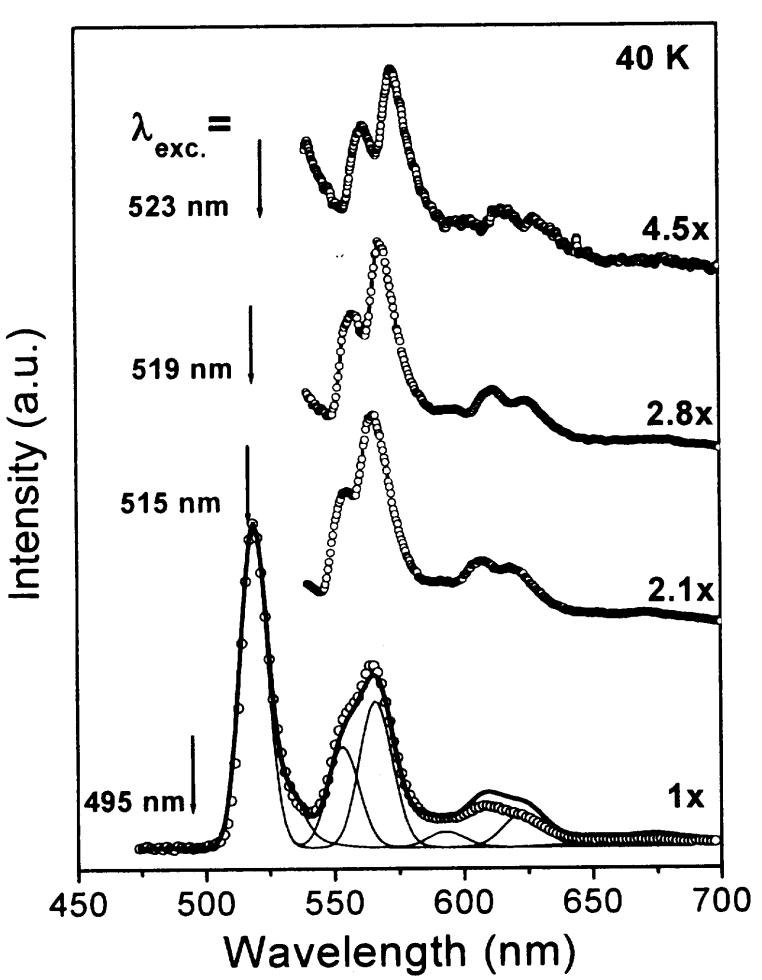

Figure 1. Dependence of SSL spectra on $\lambda_{e x c}$ (arrows). The bottom spectrum shows a full spectral line shape (circle) and its theoretical fit through Frank-Condon analysis (line).

a narrow zero-phonon line at $521 \mathrm{~nm}$ followed by a vibronic progression consisting of least three overtones, which are the combination of different effective backbone stretching modes [3]. Once $\lambda_{e x c}$ moves into resonance with the zerophonon line (from 515 to $523 \mathrm{~nm}$ ), only long (low energy) 
conjugated segments are excited and line narrowing occurs. As a consequence, the dominant unresolved vibronic band around $576 \mathrm{~nm}$ splits into a doublet. Using Franck-Condon analysis [3] for the bottom spectrum of Fig.1, we notice that the vibronic progression can be well described (continuous curve) by the overlapping of three effective, inhomogeneous broadened phonon modes with energies 330, 1160 and $1550 \mathrm{~cm}^{-1}$. The last two modes coincide with the splitted structure observed in the resonant case and are assigned to stretching vibration of vinyl group and phenyl ring $[3,4]$. The feature around $330 \mathrm{~cm}^{-1}$ has been associated to low frequency out-of-plane torsional modes [4].

We used the well-resolved vibronic modes and FranckCondon analysis to study the dependence of electronphonon coupling on the thermal disorder. Fig. 2a shows the SSL spectra $\left(\lambda_{e x c}=523 \mathrm{~nm}\right)$ in the region of vibronic progressions for different temperatures varying from 40 to 120 $\mathrm{K}$. In this temperature range there are no significant spectral shift and line broadening. This is of particular importance in the next analysis since the only varying parameters used in the spectral fitting were the Huang-Rhys factors $S_{i}$ (i=1,2,3), i.e., the coupling constants for the modes with energies 330, 1160 and $1550 \mathrm{~cm}^{-1}$, respectively. The decrease of the spectral intensity is related to non-radiactive processes activated by temperature and it was not essential in the present analysis.

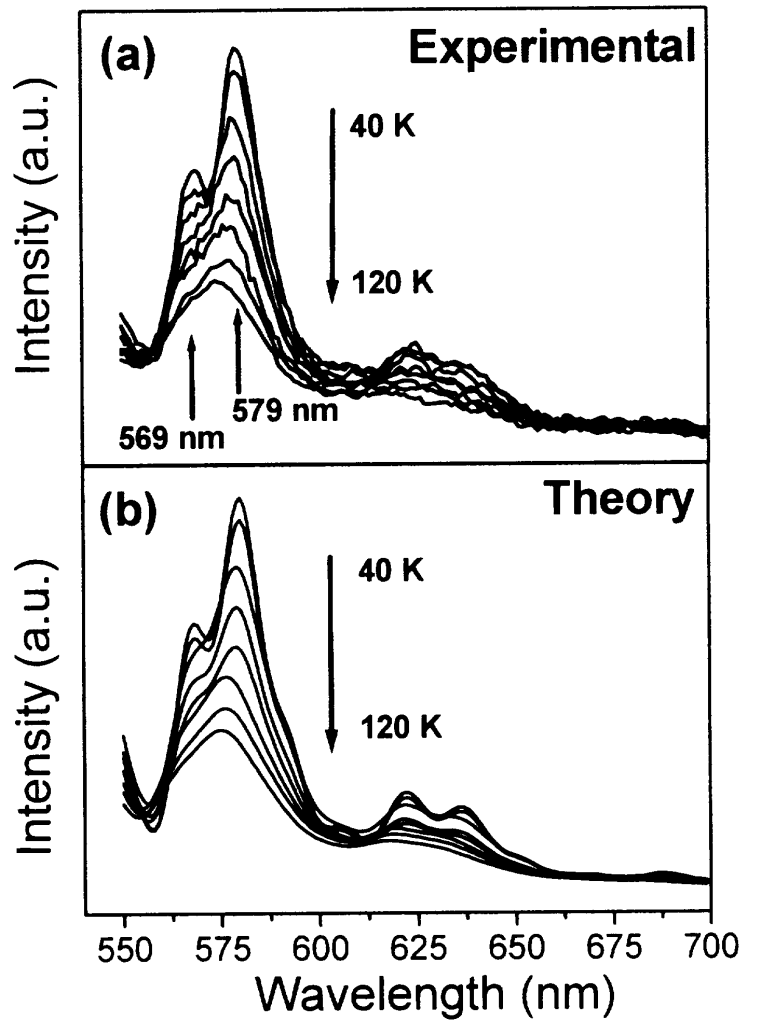

Figure 2. Temperature dependence of SSL spectra in the region of vibronic progression (a) and their theoretical fitting using FrankCondon analysis (b).

Figure $2 \mathrm{~b}$ shows the theoretical spectra obtained from a fit of those in Fig. 2a. The result thus calculated fits the experimental data quite well. It has to be remarked that the strong changes in the spectral shape observed in such narrow temperature range can be mainly accounted for the change of the electron-phonon coupling given by the $S_{i}$ factors. The temperature dependence of each $S_{i}$ obtained from the fitting is displayed in Fig. 3. One observes that the coupling constant increases with temperature for the two less energetic modes (330 and $1160 \mathrm{~cm}^{-1}$ ). This trend is consistent with the fact that $S_{j}$ is a decreasing function of conjugation length $n$. Since disorder decreases $n$, one expects that the higher the thermal disorder of a system, the larger the $S$ factor. Conversely, the higher energy mode (1160 $\mathrm{cm}^{-1}$ ) presented an almost constant behaviour in the temperature range. Both behaviour can be well explained by a n-dependent $S_{i}$ relation of the form $S_{i}=S_{i 0}+a_{i} /(n+1)$ [3], $\mathrm{S}_{i 0}$ is the $\mathrm{S}$-factor at $\mathrm{T}=0 \mathrm{~K}$ and $\mathrm{a}_{i}$ is an adjustable parameter. The temperature dependence of the conjugation length is given by $\mathrm{n}=\mathrm{n}_{o} \exp (-\varepsilon / \mathrm{kT})$ [1], where $\varepsilon$ is a characteristic energy of the conformational process and $\mathrm{n}_{o}$ is the effective conjugation length at $\mathrm{t}=0 \mathrm{~K}$. The value $\mathrm{n}_{o}=7$ was determined from temperature dependence of the energy difference between HOMO-LUMO states. From the data of Fig. 3 one find same value of $\varepsilon(=39 \mathrm{meV})$ for all phonon modes, which is in the range of the torsional barrier for polyenes [2].

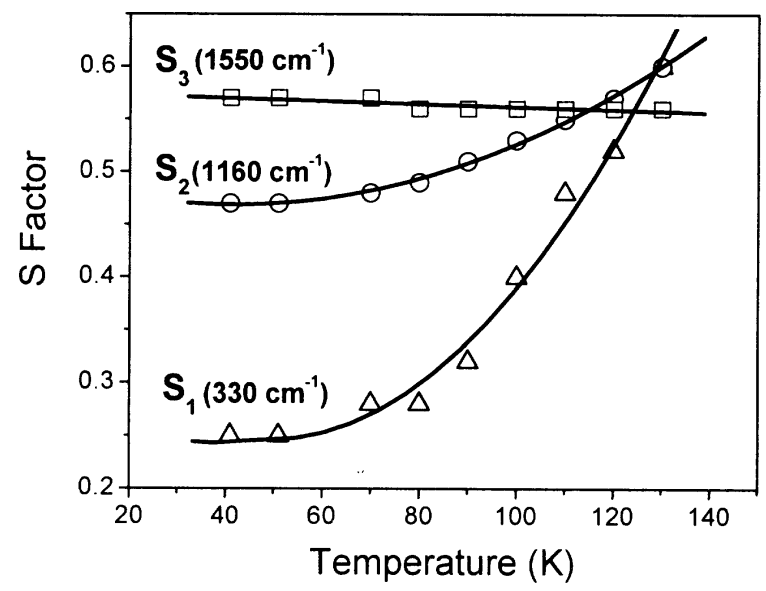

Figure 3. Temperature dependence of the $S_{i}(i=1,2,3)$ factor for the effective vibrational modes $\left(300,1160\right.$ and $\left.1550 \mathrm{~cm}^{-1}\right)$. The continuous lines depict the expected theoretical behaviors for each $\mathrm{S}_{i}$

\section{Conclusion}

In the present work we find that the Huang-Rhys factor of each mode increases differently with the temperature. We can regard as a result that the main changes of the luminescence spectra with temperature is accounted mainly for the variation of the electron-phonon coupling of the low frequency torsional modes.

\section{Acknowledgement}

The financial assistance of FAPESP and CNPq is acknowledged. 


\section{References}

[1] S. N. Yaliraki, R. J. Silbey, J. Chem. Phys. 104, 1245 (1996).

[2] P. Wood, D. W. Samuel, R. Schrock, and R. L. Christensen, J. Chem. Phys. 115, 10955 (2001).

[3] R. Chang, J. H. Hsu, W. S. Fann, K. K. Liang, C. H. Chang, M. Hayashi, J. Yu, S. H. Lin, E. C. Chang, K. R. Chuang, and S. A. Chen, Chem. Phys. Lett. 317, 142 (2000) and references there in.
[4] S. Heun, et al, J. Phys.: Condens. Matter. 5, 247 (1993).

[5] A. Marletta, F. A. Castro, D. Gonçalves, O. N. Oliveira Jr., R. M. Faria, and F. E. G. Guimarães, Synth. Met. 121, 1447 (2001).

[6] A. Marletta, O.N. Oliveira Jr., R. M. Faria, and F. E. G. Guimarães, Macromolecules 33, 5886 (2000). 